\title{
Controlling the intrinsic bending of hetero-epitaxial silicon carbide micro-cantilevers
}

\author{
Atieh Ranjbar Kermany and Francesca lacopi ${ }^{\mathrm{a}}$ \\ Queensland Micro- and Nanotechnology Centre, Griffith University, Nathan, QLD 4111, \\ Australia
}

We introduce a simple methodology to predict and tailor the intrinsic bending of a cantilever made of a single thin film of hetero-epitaxial silicon carbide grown on silicon. The combination of our novel method for the depth profiling of residual stress with a few nm resolution with finite element modelling allows for the prediction of the bending behaviour with great accuracy. We also demonstrate experimentally that a silicon carbide cantilever made of one distinct film type can be engineered to obtain the desired degree of either upward, flat, or downward bending, by selecting the appropriate thickness and cantilever geometry. A precise control of cantilever bending is crucial for MEMS applications such as micro-actuators, micro-switches, and resonant sensors.

\section{INTRODUCTION}

Epitaxial cubic silicon carbide (3C-SiC) is a leading material for micro electrical mechanical systems (MEMS) due to its excellent mechanical properties when silicon (Si) has limitations. ${ }^{1-4}$ Furthermore, it can be grown on Si substrate which results in large area, easy micromachining, and low cost production. ${ }^{5,6}$ However, the difference in Si and 3C-SiC lattice constants ( $20 \%$

\footnotetext{
a) Electronic mail: f.iacopi@griffith.edu.au
} 
mismatch) and thermal expansion coefficients ( $\sim 8 \%$ mismatch during the cool down) results in wafer bow and a high residual stress. ${ }^{5,7}$

The residual stress affects the mechanical behaviour of a thin film, and their consequence could be advantageous or detrimental depending on the application. ${ }^{8}$ We have previously shown that high residual stress can improve the sensitivity parameter (frequency $(f) \times$ quality factor $(Q)$ ) of resonant microstrings, ${ }^{6}$ which could be beneficial for applications such as resonant sensing. Whereas, stress-free microstructures are required for applications such as pressure sensors to prevent the stiffening effect. ${ }^{9}$

Residual stress can also result in the deformation of released microstructures. In the case of the single-clamped structures such as a cantilever, uniform (mean) residual stress within the film would be released because of the free end. However, if there is a variation of residual stress over the film thickness (gradient stress), ${ }^{10}$ the structure will bend upon release. ${ }^{7,11-14}$ This intrinsic bending could be applied favourably in MEMS thermal actuators such as normally-closed microcages ${ }^{15}$ micro-wrappers, ${ }^{16}$ and micro-grippers, ${ }^{17,18}$ and electrostatic actuators. ${ }^{19,20}$ Similarly, the bending can greatly reduce the required voltage for MEMS switches. ${ }^{21-23}$ However, flat structures are required in applications such as atomic force microscopy (AFM), and most resonant sensors. ${ }^{24,25}$ As a result, it is important to understand, control, and engineer the intrinsic bending depending on the application.

Most literature reports have focused on the tuning of film deposition parameters to alter stress gradients, ${ }^{7,26}$ or metallization of the thin film ${ }^{8,27,28}$ in order to manipulate the intrinsic bending of the released structures. Some also used multilayer structures in order to balance and control the intrinsic bending. ${ }^{29,30}$ In this paper, we demonstrate the tailoring of the intrinsic bending of a single-layered cantilever made of hetero-epitaxial 3C-SiC on silicon by combining 
the knowledge of the film gradient stress (in nanometer $(\mathrm{nm})$ resolution) ${ }^{31}$ with finite element modelling (FEM). The stress gradient of a hetero-epitaxial 3C-SiC film is so complex that we can engineer 3C-SiC cantilevers to bend upward, downward, or to be nearly flat, even by just using one single film, as the result of selecting the appropriate thickness and film type. We also study the influence of the cantilever geometry and the gravity on the degree of bending.

\section{EXPERIMENT}

3C-SiC films with thickness of 200-250 nm were grown on $150 \mathrm{~mm}$ silicon wafers with $<100>$ and $<111>$ surface orientations at $1000{ }^{\circ} \mathrm{C}$ in a hot-wall horizontal low-pressure chemical vapour deposition chamber, using silane $\left(\mathrm{SiH}_{4}\right)$ and propene $\left(\mathrm{C}_{3} \mathrm{H}_{6}\right)$ as precursor gases. ${ }^{32}$ The thickness was measured using Veeco Wyko NT1100 optical profilometer which has a resolution of $1 \AA$, assuming a constant refractive index of 2.65 for $3 \mathrm{C}$-SiC films. ${ }^{33}$ The stresses were calculated from the wafer warpage measured with a Tencor Flexus 2320 system. The gradient stresses throughout the SiC films thickness were calculated over monitoring the wafer curvature by etching back the films through sequential steps of $\sim 10-20 \mathrm{~nm}$ thickness, as detailed in our previous work. ${ }^{31}$ The Young's modulus $(E)$ was calculated using Hysitron Triboindenter nanoindentation on $1 \mu \mathrm{m}$ thick films. It should be noted that $E$ might be affected by the defect density and so it is not necessarily uniform throughout the thickness. ${ }^{34-36}$ With the assumption that the Young's modulus is constant throughout the thickness, we calculated values of $400 \mathrm{GPa}$

and $330 \mathrm{GPa}$ for $\mathrm{SiC}(111)$ and $\operatorname{SiC}(100)$ respectively. ${ }^{32}$ As the result of this assumption, the residual stress values might be a bit overestimated.

The different SiC film thicknesses studied in this work were obtained by etching back the 250 thick films after their deposition. For this purpose, we used an inductively coupled plasma (ICP) STS 
anisotropic etcher with a flow of $50 \mathrm{sccm}$ hydrogen chloride $(\mathrm{HCl})$ at 4 mTorr and room temperature to perform the etching. SiC thickness uniformity remained around $2 \%$ with $3-5 \mathrm{~nm}$ variation across the $150 \mathrm{~mm}$ wafer. ${ }^{37}$

We fabricated the cantilevers using Si surface micromachining and through the four stages of photolithography, SiC etching (STS etcher), Si etching (using xenon difluoride $\left(\mathrm{XeF}_{2}\right)$ isotropic dry chemical etcher) and photoresist removal (using TEGAL 915 oxygen plasma with $1200 \mathrm{mTorr}$ pressure, $100^{\circ} \mathrm{C}$ temperature, and 550W RF power). We used two photolithography steps in order to release the structures perfectly-clamped. We used the first lithography step to develop the cantilever patterns on SiC film. Subsequently, we used the second lithography step after the SiC etching and prior to the Si etching in order to protect the cantilevers anchors from overetching due to the isotropic behaviour of the $\mathrm{XeF}_{2}$ etching process. The details on perfectlyclamped fabrication method can be found in our previous work. ${ }^{6}$

We analysed the intrinsic bending by comparing the finite element modelling (FEM) analysis to the scanning electron microscopy (SEM) micrographs of experimentally fabricated cantilevers. The SEM was performed with a JEOL JSM-6510 LV. We performed the FEM analysis using the IntelliSuite software (version 8.7). The cantilevers were designed and their intrinsic bending was analysed using the 3DBuilder and the Thermo-Electro-Mechanical (TEM) modules of the software. The Young's modulus, Poisson’s ratio ( $v)$, density $(\rho)$, and residual were inserted in the TEM module for the analysis. The Poisson's ratio values of 0.235 for $\mathrm{SiC}(111)$ and 0.267 for $\mathrm{SiC}(100)$ and density of $3.21 \mathrm{gcm}^{-2}$ were taken from literature. ${ }^{3,38}$ The SiC residual stress was input by subdividing the modelled 3C-SiC films into multiple nm thick layers. The stress of each thin layer was assigned according to the layer-by-layer stress profiles of our recent work ${ }^{31}$ (FIG.1). 


\section{RESULTS AND DISCUSSION}

\section{A. Stress relaxation mechanism upon growth}

The epitaxial growth of 3C-SiC on silicon typically leads to high residual tensile film stresses, due to a combination of lattice and thermal mismatches. The stress arising from the thermal mismatch is not expected to contribute to stress gradients, as it is only related to the cooling phase after the film growth., ${ }^{3,10,12}$ The overall film residual mean stress, as well as the stress gradient behaviour and resulting defect densities, depend strongly on the chosen deposition conditions, as they are directly linked to the stress relaxation rates upon film growth. ${ }^{10}$

Even though the strain produced from the lattice and thermal mismatches can be considered independent of the Si orientation, we have reported that the relaxation over thickness (gradient stress) is faster in $\mathrm{SiC}(100)$ as compared to $\mathrm{SiC}(111)$ when using the same film growth process. ${ }^{31}$ This is due to the fact that the density of the stacking faults (SFs), which are one of the dominant stress relaxation mechanisms in 3C-SiC films, ${ }^{12,37}$ are more efficient in $\mathrm{SiC}(100)$ films because of their more favourable geometrical projection onto the $<100>$ Si growth plane. $^{31,32}$

Figure 1(a) and 1(b) show a typical stress gradient depth profile for the $\operatorname{SiC}(100)$ and $\mathrm{SiC}(111)$ films, respectively, measured with high resolution as discussed in our previous work. ${ }^{31}$ Both SiC films show a highly compressed nanolayer at the interface with the substrate, which we had attributed to the out-diffusion of silicon in the carbonization layer. ${ }^{31}$ This could also be linked to the super cell mismatch between $\mathrm{SiC}$ and Si pointed out by Zielinski et al. ${ }^{10}$ As the growth proceeds, both films quickly build up a tensile stress due to the thermal mismatch contribution. This results in a sharp positive (tensile) gradient. This tensile gradient continues until both films reach about $500 \mathrm{MPa}$ of tensile stress, around 20-30nm thickness. Interestingly, while the $\mathrm{SiC}(111)$ film retains that value of tensile stress throughout the remaining thickness, as 
thickness increases beyond $\sim 35 \mathrm{~nm}$, the $\mathrm{SiC}(100)$ film stress starts to relax resulting in a negative (compressive) gradient. The stress of the topmost layers of the $\mathrm{SiC}(100)$ film evolve towards full relaxation (stress value approaching $100 \mathrm{MPa}$ ). Consequently, the stress gradient behaviour versus thickness can be divided to two regions, a highly tensile followed by a slightly compressive region for the $\mathrm{SiC}(100)$ film, and a highly tensile region followed by an almost flat plateau for the $\mathrm{SiC}(111)$ film, as shown in Fig. 1(a) and 1(b), respectively. Note that this analysis was limited to relatively thin SiC films; larger thicknesses are required to further examine the relaxation rate in $\mathrm{SiC}(111)$ film.
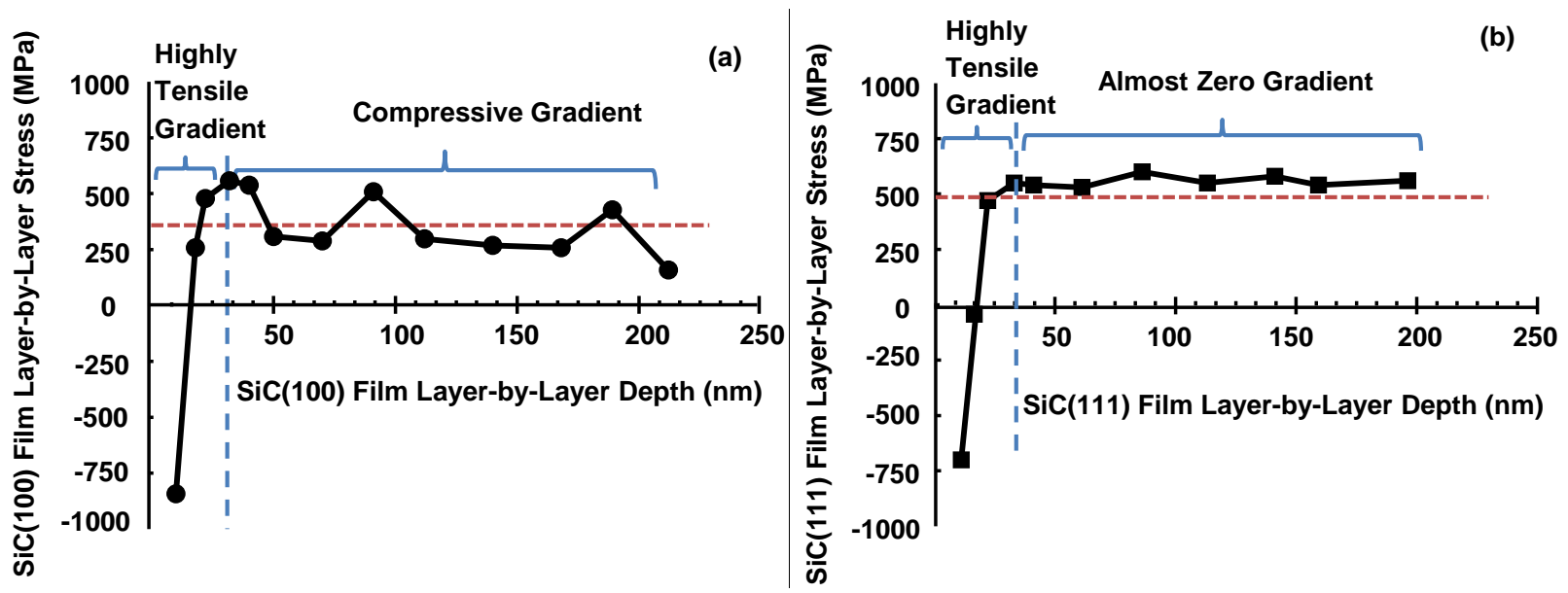

FIG. 1. Layer-by-layer Stress profile; stress gradient regions for on-axis (a) $\operatorname{SiC}(100)$ and (b) $\mathrm{SiC}(111) .{ }^{31} \mathrm{SiC}(100)$ have a tensile gradient and a compressive gradient region above $\sim 35 \mathrm{~nm}$ thickness, resulting from film relaxation, while the $\operatorname{SiC}(111)$ shares the same initial tensile gradient, but shows a nearly flat behaviour (nearly zero gradient) thereafter. The red dotted line is added only to guide the eye.

In a single-layer cantilever, the stress gradients within the single layer are responsible for the intrinsic bending. Thus, the direction of the cantilever bending is influenced by the gradient stress state. 
For the $\mathrm{SiC}(100)$ films, we observe upward bending for thicknesses up to $\sim 77 \mathrm{~nm}$ because the dominant gradient stress is tensile. Figure 2(a) and 2(b) show FEM and SEM results respectively, obtained for a $\mathrm{SiC}(100)$ cantilever with the thickness of $66 \mathrm{~nm}$. The FEM image (Fig. 2(a)) shows an upward deflection of $\sim 60 \mu \mathrm{m}$. The value is in good agreement with the fabrication result (Fig 2(b)), validating the model based on the measured gradient data.

At $77 \mathrm{~nm}$ thickness, the FEM analysis predicts nearly zero bending for the $\mathrm{SiC}(100)$ cantilever. At this point, the compressive and tensile stress gradients are almost equal and so the cantilever is flat. Indeed, for thicknesses slightly above $77 \mathrm{~nm}$, we start observing experimentally downward bending for the $\mathrm{SiC}(100)$ cantilever. Figure 2(c) and 2(d) show the FEM analysis and an SEM image of a SiC(100) cantilever with the thickness of $80 \mathrm{~nm}$. The SEM image (Fig. 2(d)) shows slightly downwards bending which is in good agreement with the FEM result (bending of $\sim 3 \mu \mathrm{m})$. The downward bending happens due to the fact that the dominant gradient starts to be the compressive one.

The degree of downward bending increases as thickness increases to $250 \mathrm{~nm}$ because the compressive gradient stress increases. Figure 2(e) and 2(f) are FEM and SEM images of a SiC(100) cantilever with the thickness of $250 \mathrm{~nm}$. A negative bending of $\sim 60 \mu \mathrm{m}$ can be observed through the FEM result (Fig. 2(e)), which is again in good agreement with the fabrication result (Fig. 2(f)).

As for the $\mathrm{SiC}(111)$ cantilever, the bending behaviour is similar to the $\mathrm{SiC}(100)$ cantilever for the highly tensile region. In addition, the bending remains upward for any thickness up to the maximum investigated here because the stress gradient remains tensile throughout. Figure 3(a) and 3(b) show the deflection comparison between $\operatorname{SiC}(100)$ and $\operatorname{SiC}(111)$ cantilevers with 250 nm thickness. 


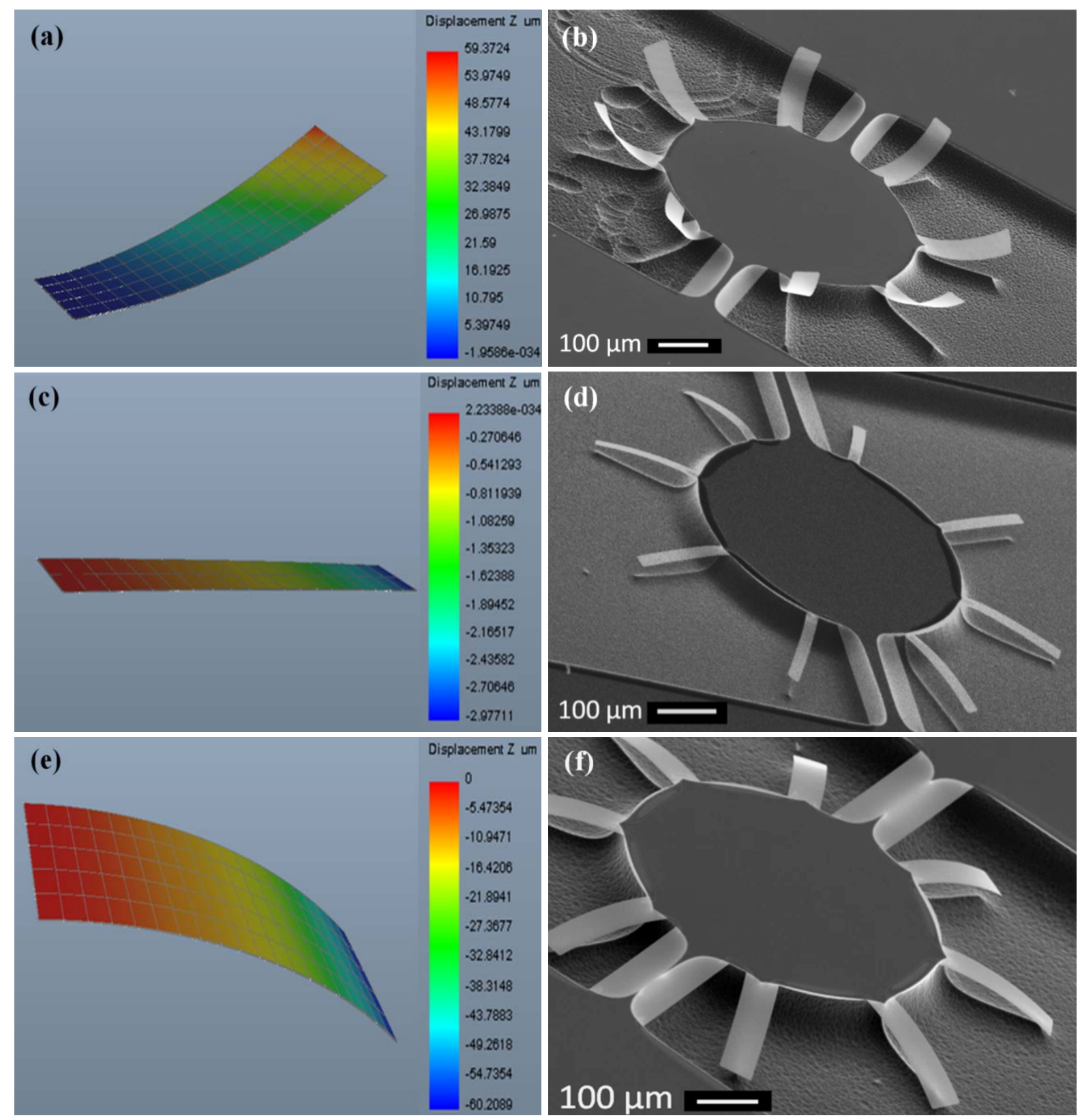

FIG. 2. SiC(100) cantilevers with $200 \mu$ m length bending behaviour: upward bending due to the tensile gradient region (a) FEM and (b) SEM results (50 $\mu$ m width, and $66 \mathrm{~nm}$ thickness); nearly flat region (c) FEM and (d) SEM images (20 $\mu \mathrm{m}$ width, and $80 \mathrm{~nm}$ thickness); and downward bending due to the compressive gradient region (e) FEM and (f) SEM results (50 $\mu \mathrm{m}$ width, and $250 \mathrm{~nm}$ thickness). The SEM images are taken with $60^{\circ}$ tilting. 

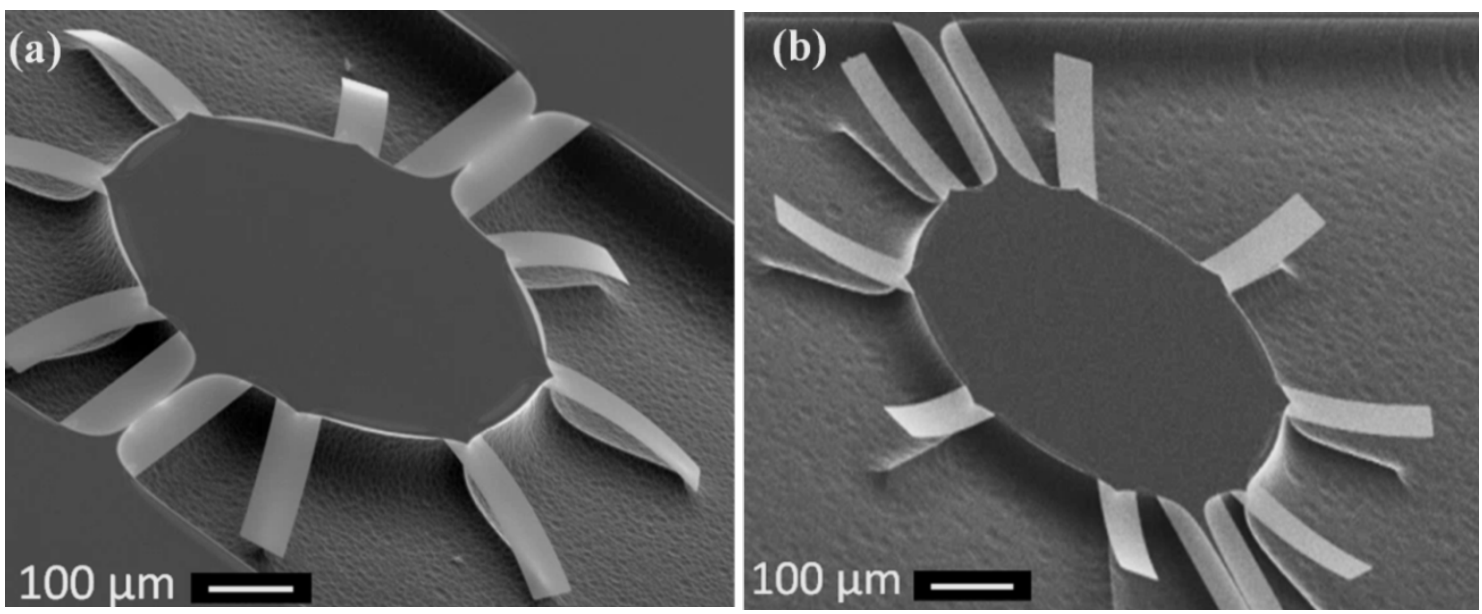

FIG. 3. Intrinsic bending comparison of cantilevers made of (a) SiC(100) and (b) SiC(111), with length of $200 \mu \mathrm{m}$, width of $50 \mu \mathrm{m}$, and thickness of $250 \mathrm{~nm}$. The SEM images are taken with $60^{\circ}$ tilting.

Overall, we observe upward, flat, and downward intrinsic bending in $\mathrm{SiC}(100)$ cantilever but only upward bending for SiC(111) cantilever for thicknesses up to $250 \mathrm{~nm}$, due to the difference in their stress relaxation rate. Consequently, engineering the thin film cantilever bending to three forms of upward, flat and downward is only possible through the $<100>$ orientation.

Note that this finding is potentially in agreement with the work by Zielinski and coworkers. $^{39-41}$ They investigated cantilever bending for SiC with thicknesses from $100 \mathrm{~nm}$ to above $1 \mu \mathrm{m}$ and shown constant upward bending in SiC(111) as we report, but only downward bending in $\mathrm{SiC}(100)$. As we show here, the upward bending of $\mathrm{SiC}(100)$ is restricted to a narrow region, i.e. films thinner than $80 \mathrm{~nm}$. In the light of our findings, it is likely that upward bending would have been observed only for films thinner than those they have analysed.

\section{B. Cantilever geometry}

The degree of bending can be further influenced by the cantilever geometry and the 3C-SiC elastic properties. The maximum vertical deflection $(\delta)$ of the cantilever can be described by ${ }^{42}$ 


$$
\delta=\frac{M(1-v) l^{2}}{2 E I},
$$

Where $M$ is the bending moment and $l$ is the cantilever length. $I$ is the inertia momentum (rectangular cross section: $I=\left(w t^{3}\right) / 12$, where $w$ and $t$ are the cantilever width and thickness respectively). ${ }^{7,25}$ When assuming a linear stress gradient, $M=\sigma_{1} I$ can be used, and therefore, the effect of the cantilever width can be neglected. ${ }^{42}$

We can see from Eq. (2) that the vertical deflection is directly affected by the cantilever length $(l){ }^{14,43-45}$ This is also evident in Fig. 4 . Thus, the cantilever geometry affects the degree of upward or downward bending but it does not initiate the deflection. As mentioned, the origin of the cantilever intrinsic bending is the stress gradient.

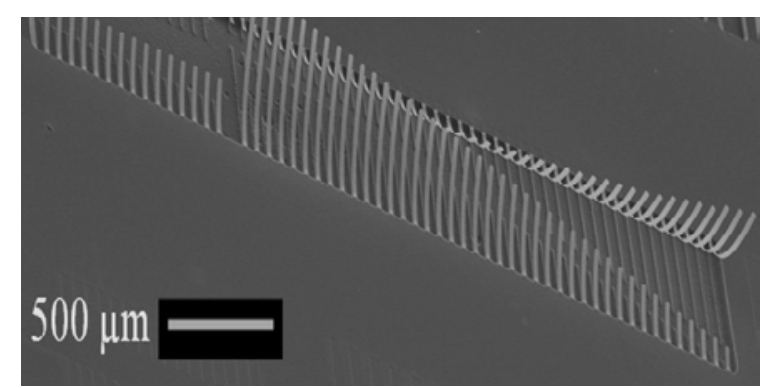

FIG. 4. SEM image of SiC(111) cantilevers with varying length (100 $\mu \mathrm{m}$ to $1000 \mu \mathrm{m})$, width of $20 \mu \mathrm{m}$, and thickness of $250 \mathrm{~nm}$. The SEM image is taken with $60^{\circ}$ tilting.

\section{Gravity}

The degree of bending can also be influenced by the gravity. We calculated the vertical deflection of the 3C-SiC cantilever (with different geometries) due to gravity through the equation $^{7}$

$$
\xi=\frac{w t \rho l^{4}}{8 E I}
$$


Where $\xi$ is the cantilever deflection due to the gravity. We find the cantilevers maximum deflection due to the gravity in small picometers (pm) ranges. Consequently, the gravity effect on the 3C-SiC cantilever vertical deflection can be ignored.

\section{CONCLUSION}

This work brings important insights on the cantilever intrinsic bending behaviour. We demonstrated a simple method to predict, control and engineer the intrinsic bending of a singlelayered 3C-SiC cantilever by selecting the appropriate film type, thickness and cantilever length. We predicted the cantilever intrinsic bending behaviour with great accuracy through the FEM simulation combined with the measured film stress gradient (in $\mathrm{nm}$ resolution). In addition, we confirmed experimentally that upward, flat, or downward bending with desired degrees could be obtained for $\mathrm{SiC}(100)$ cantilevers by selecting the thickness in the appropriate regions. We also confirmed that the degree of bending can be further adjusted by varying the cantilever length. This way we can tailor the bending direction and degree to the targeted application. Deflected cantilevers with desired bending degrees can be used for MEMS applications such as microactuators and micro-switches whereas flat cantilever is mainly preferred for resonant sensors.

\section{ACKNOWLEDGEMENT}

Fabrication was performed using the equipment of the Australian National Fabrication Facilities (ANFF), Queensland node. We thank Marcin Zielinski from NovaSiC for helpful discussion and Neeraj Mishra from Griffith University for experimental suppor. The authors acknowledge the support from AFOSR through the AOARD 15IOA053 grant. Assoc. Prof. F. Iacopi is the recipient of an Australian Research Council Future Fellowship (FT120100445). 
1 N. G. Wright and A. B. Horsfall, J. Phys. D: Appl. Phys. 40 (20), 6345 (2007).

2 C. A. Zorman and R. J. Parro, Phys. Stat. Sol. (b) 245 (7), 1404 (2008).

3 V. Cimalla, J. Pezoldt, and O. Ambacher, J. Phys. D: Appl. Phys. 40 (20), 6386 (2007).

4 B. V. Cunning, M. Ahmed, N. Mishra, A. R. Kermany, B. Wood, and F. Iacopi, Nanotech. 25 (32), 325301 (2014).

5 A. Severino, C. Locke, R. Anzalone, M. Camarda, N. Piluso, A. La Magna, S. Saddow, G. Abbondanza, G. D'Arrigo, and F. La Via, ECS Trans. 35 (6), 99 (2011).

${ }^{6}$ A. R. Kermany, G. Brawley, N. Mishra, E. Sheridan, W. P. Bowen, and F. Iacopi, Appl. Phys. Lett. 104 (8), 081901 (2014).

7 R. Anzalone, M. Camarda, C. Locke, D. Alquier, A. Severino, M. Italia, D. Rodilosso, C. Tringali, A. La Magna, and G. Foti, J. Electrochem. Soc. 157 (4), H438 (2010).

8 J. Pezoldt, R. Nader, F. Niebelschütz, V. Cimalla, T. Stauden, C. Zgheib, and P. Masri, Phys. Stat. Sol. (a) 205 (4), 867 (2008).

9 F. Niebelschütz, K. Brueckner, V. Cimalla, M. A. Hein, and J. Pezoldt, Mater. Sci. Forum 615-617, 621 (2009).

${ }^{10}$ M. Zielinski, A. Leycuras, S. Ndiaye, and T. Chassagne, Appl. Phys. Lett. 89, 131906 (2006).

${ }^{11}$ R. Anzalone, G. D'arrigo, M. Camarda, C. Locke, S. E. Saddow, and F. La Via, J. Microelectromech. Syst. 20 (3), 745 (2011).

${ }^{12}$ M. Bosi, B. E. Watts, G. Attolini, C. Ferrari, C. Frigeri, G. Salviati, A. Poggi, F. Mancarella, A. Roncaglia, and O. Martínez, Cryst. Growth Des. 9 (11), 4852 (2009).

${ }^{13}$ B. Van Drieënhuizen, J. Goosen, P. French, and R. Wolffenbuttel, Sens. Actuators A: Phys. 37, 756 (1993). 
${ }^{14}$ M. Lishchynska, N. Cordero, O. Slattery, and C. O'Mahony, J. Micromech. Microeng. 15 (7), S10 (2005).

${ }^{15}$ J. K. Luo, R. Huang, J. H. He, Y. Q. Fu, A. J. Flewitt, S. M. Spearing, N. A. Fleck, and W. I. Milne, Sens. Actuators A: Phys. 132 (1), 346 (2006).

${ }^{16}$ J. Gill, D. T. Chang, L. A. Momoda, and G. P. Carman, Sens. Actuators A: Phys. 93 (2), 148 (2001).

${ }^{17}$ Y. Bellouard, Mater. Sci. Eng., A 481, 582 (2008).

${ }^{18}$ Y. Fu, H. Du, W. Huang, S. Zhang, and M. Hu, Sens. Actuators A: Phys. 112 (2), 395 (2004).

${ }^{19}$ J. Musolf and P. Kohl, U.S. patent 6,625,004 (23 September 2003).

${ }^{20}$ G. D. Gray, M. J. Morgan, and P. A. Kohl, in MEMS Components and Applications for Industry, Automobiles, Aerospace, and Communication II (Proc. SPIE 2003), Vol. 4981, pp. 202.

${ }^{21}$ P. Schmid, F. J. Hernandez-Guillen, and E. Kohn, Diamond Relat. Mater. 12 (3), 418 (2003).

${ }^{22}$ R. T. Chen, H. Nguyen, and M. C. Wu, IEEE Photon. Technol. Lett. 11 (11), 1396 (1999).

${ }^{23}$ D. Peroulis, K. Sarabandi, and L. Katehi, in Microwave Symposium Digest, 2002 IEEE MTT-S International (IEEE, Seattle, WA, USA, 2002), Vol. 1, pp. 223.

${ }^{24}$ N. V. Lavrik, M. J. Sepaniak, and P. G. Datskos, Rev. Sci. Instrum. 75 (7), 2229 (2004).

${ }^{25}$ A. Boisen, S. Dohn, S. S. Keller, S. Schmid, and M. Tenje, Rep. Prog. Phys. 74 (3), 036101 (2011).

${ }^{26}$ F. J. Hernandez-Guillen, K. Janischowsky, J. Kusterer, W. Ebert, and E. Kohn, Diamond Relat. Mater. 14 (3), 411 (2005).

${ }^{27}$ C. Zgheib, P. M. Masri, P. Weih, O. Ambacher, and J. Pezoldt, Mater. Sci. Forum 457-460, 301 (2004). 
${ }^{28}$ J. Pezoldt, T. Stauden, F. Niebelschütz, M. A. Alsioufy, R. Nader, and P. M. Masri, Mater. Sci. Forum 645-648, 159 (2010).

${ }^{29}$ M. A. Matin, K. Ozaki, D. Akai, K. Sawada, and M. Ishida, Comput. Mater. Sci. 85, 253 (2014).

${ }^{30}$ L. Wen, H. Zeng, Z. Yuan, J. Chu, and H. Wang, in Nano/Micro Engineered and Molecular Systems (NEMS), 2011 IEEE International Conference on (IEEE, 2011), pp. 634.

${ }^{31}$ F. Iacopi, R. E. Brock, A. Iacopi, L. Hold, and R. H. Dauskardt, Acta Mater. 61 (17), 6533 (2013).

${ }^{32}$ F. Iacopi, G. Walker, L. Wang, L. Malesys, S. Ma, B. V. Cunning, and A. Iacopi, Appl. Phys. Lett. 102 (1), 011908 (2013).

${ }^{33}$ S. Singh, J. R. Potopowicz, L. G. Van Uitert, and S. H. Wemple, Appl. Phys. Lett. 19 (3), 53 (1971).

${ }^{34}$ R. Anzalone, M. Camarda, A. Canino, N. Piluso, F. La Via, and G. D’Arrigo, Electrochem. Solid-State Lett. 14 (4), H161 (2011).

${ }^{35}$ B. Hähnlein, M. Stubenrauch, S. Michael, and J. Pezoldt, Mater. Sci. Forum 778, 444 (2014).

${ }^{36}$ B. Hähnlein, M. Stubenrauch, and J. Pezoldt, Mater. Sci. Forum 821, 281 (2015).

${ }^{37}$ N. Mishra, L. Hold, A. Iacopi, B. Gupta, N. Motta, and F. Iacopi, J. Appl. Phys. 115 (20), 203501 (2014).

${ }^{38}$ H. Yao, L. Ouyang, and W. Y. Ching, J. Am. Ceram. Soc. 90 (10), 3194 (2007).

${ }^{39}$ M. Zielinski, J. F. Michaud, S Jiao, T. Chassagne, A. E. Bazin, A. Michon, M. Portail, and D. Alquier, Mater. Sci. Forum 679, 79 (2011).

${ }^{40}$ S. Jiao, M. Zielinski, J. F. Michaud, T. Chassagne, M. Portail, and D. Alquier, Mater. Sci. Forum 711, 84 (2012). 
${ }^{41}$ M. Zielinski, J. F. Michaud, S. Jiao, T. Chassagne, A. E. Bazin, A. Michon, M. Portail, and D. Alquier, J. Appl. Phys. 111, 053507 (2012).

${ }^{42}$ A. Ballestra, A. Somà, and R. Pavanello, Sensors 8 (2), 767 (2008).

${ }^{43}$ M. Camarda, R. Anzalone, A. Severino, N. Piluso, A. La Magna, and F. La Via, Mater. Sci. Forum 679-680, 213 (2011).

${ }^{44}$ M. Camarda, R. Anzalone, A. Severino, N. Piluso, A. Canino, F. La Via, and A. La Magna, J. Mater. Res. 28 (01), 104 (2013).

${ }^{45}$ W. Fang, C. Lee, and H. Hu, J. Micromech. Microeng. 9 (3), 236 (1999). 